\section{Population research and mental health policy}

\author{
Bridging the gap
}

\author{
BRIAN COOPER and BRUCE SINGH
}

Twenty years ago a World Psychiatric Association (WPA) international symposium under the title 'What is a case?' (Wing et al, 1981) served to highlight a central methodological problem of psychiatric epidemiology. Since then, incorporation in the main classification systems of glossaries and operational guidelines (World Health Organization, 1993; American Psychiatric Association, 1994), together with advances in standardised interview techniques, seemed to have so far underpinned diagnostic assessment that this issue, if not fully resolved, had at any rate lost much of its immediacy. However, application of the concept of 'medical necessity' in managedcare programmes (Ford, 1998) has led to a critical reappraisal in the USA. Regier $e t$ al (1998), in a review of estimates from two large-scale USA projects, the Epidemiologic Catchment Area (ECA) studies and the National Comorbidity Survey (NCS), noted that these differed widely. Thus, for the age range $18-54$ years, the mean annual percentage prevalence rates for alcohol dependence, major depressive disorder, anxiety disorder, panic disorder and social phobia were 4.1, 4.2, 9.9, 1.1 and 1.6, respectively, in the ECA studies (one-wave estimates) and 7.4, 10.1, 15.3, 2.2 and 7.4, respectively, in the NCS. Corresponding differences were found between 'lifetime' prevalence rates for these categories. Such large disparities, in estimates derived by similar methods in the same country only a few years apart, are unlikely to be due to real differences in morbidity experience, and must raise doubts about the accuracy of case reporting.

Apart from diagnostic inconsistency, total prevalence rates in the two projects were deemed too high to provide useful guidance to the extent of need as gauged by medical necessity. In the ECA first wave, one-year psychiatric prevalence was $20 \%$ and lifetime prevalence $32 \%$, and following a second wave of interviewing these figures rose to $28 \%$ and $44 \%$, respectively. The single-wave NCS yielded for the age range 18-54 years a mean one-year prevalence of $29 \%$ and a lifetime prevalence of $48 \%$. All these rates are so much higher than the annual estimate of $2.8 \%$ for severe mental disorders in the USA (National Advisory Mental Health Council, 1993) as to raise doubts about the relevance of such data in gauging service needs. Regier et al (1998) considered it unlikely, given current USA preoccupation with cost-containment and managed-care plans, that mental health care could be provided for $28 \%$ or $29 \%$ of the population in any one year, and went on to speculate that in many of the reported cases this might not in fact be required:

\footnotetext{
". . . It is possible that many people with currently defined mental syndromes (in particular among the affective and anxiety disorders) not brought to medical attention may be having appropriate homeostatic responses that are neither pathologic nor in need of treatment" (Regier et al, 1998).
}

These remarks, coming from workers who themselves were involved in planning and coordinating the research projects in question, deserve to be taken seriously, particularly in view of current plans for a World Mental Health (WMH 2000) Initiative, involving a series of parallel community surveys in countries across the globe (Kessler, 1999). If one accepts that prevalence statistics are of limited value in causal inquiry, and that the main value of cross-sectional surveys (other than as a baseline for prospective studies) lies in providing the descriptive statistics required for health care policy and planning, it follows that their findings must be capable of evaluation within that frame of reference. The broad argument, however, condenses a number of distinct points: (a) that the validity of case identification and diagnosis in community surveys still needs improvement; (b) that such research should provide information on unmet needs for specialist treatment and care; and (c) that there are wider implications for health policy if a high prevalence of clinically significant disorders - including milder forms - is indeed confirmed.

\section{DIAGNOSTIC VALIDITY IN FIELD STUDIES}

The historical background has been reviewed by Dohrenwend (1990), and more recent developments by Brugha et al (1999a). In first-generation surveys prior to the Second World War, most investigators relied on key informants and agency records, while the handful of studies based on direct (unstructured) interviewing by psychiatrists yielded low prevalence estimates, with a mean of under $4 \%$. From the Second World War to around 1980, many secondgeneration surveys used clinical assessment by psychiatrists, based on personal examination or evaluation of interview protocols, and sometimes on collateral information. A number of standardised interviews, such as the Psychiatric Status Schedule (PSS; Spitzer et al, 1970) and the Present State Examination (PSE; Wing et al, 1974) were developed and found on testing to provide good interrater agreement. A review of research in this period revealed a general increase in reported prevalence, the average being now around 20\% (Dohrenwend \& Dohrenwend, 1974).

In the third-generation period of the past 20 years, reported case rates have further increased, as the above-cited ECA and NCS survey data suggest. During these years there has been a shift away from expert assessment, towards reliance on lay interviewers and questionnaire techniques: a trend encouraged by the use of lifetime measures, derived from self-reporting of symptoms at any time in the past. Semi-structured or guided interviews have been largely replaced by tightly structured schedules with closed questions, and clinical evaluation by computerised diagnosis. Such techniques can be, and have been, applied to large population samples (in some instances upward of 10000 persons) but must, by virtue of the interviewers' lack of experience, omit the elements of flexible probing and of clinical judgement in interpreting responses, previously thought necessary for diagnosis. The best-known packages, the National Institute of Mental Health (NIMH) Diagnostic Interview Schedule (DIS; Robins et al, 1981) and its successor, the Composite International Diagnostic Interview (CIDI; Robins et al, 1988), were developed explicitly as a response to the need for an instrument that 
would as closely as possible replicate a psychiatrist's diagnosis in situations where diagnosis by clinicians was not feasible (Helzer $e t$ al, 1985; Kessler, 1999).

The same practical necessity has decreed that a number of instruments originally intended for clinicians should either by adapted for use by lay interviewers (Rodgers \& Mann, 1986; Lewis et al, 1992), or else incorporated into fullystructured schedules (Robins et al, 1988). Even the most comprehensive 'clinical' system, the Schedules for Clinical Assessment in Neuropsychiatry (SCAN; Wing et al, 1990) in its survey format (SCAN-SF) is now being administered by lay interviewers, following a four-week training period (Brugha et al, 1999b). One consequence of the general trend has been a marked divergence between survey assessments and those made when psychiatrists undertake a clinical reappraisal of the same individuals (Anthony et al, 1985; Helzer et al, 1985; Kendler et al, 1996). This lack of congruence strongly suggests that reliance on fully-standardised survey techniques has not in fact settled the question of diagnostic validity.

What steps can be taken so that psychiatric field-studies may in future contribute more usefully to health-service planning and provision? A number of different strategies have been proposed, though it is as yet unclear what impact each of these would be likely to have in practice.

\section{Selecting the right measures of morbidity}

A remarkable feature of research in this field over the past 20 years has been its abiding preoccupation with lifetime measures of morbidity, despite their inaccuracy and the attendant problems of interpretation (Parker, 1987). Kessler (1999) points out, for instance, that the focus of almost all CIDIbased surveys to date has been on lifetime prevalence, and that to get reliable one-year estimates in the planned WMH 2000 project, a special modification of this instrument will be required. Inflation of reporting rates might therefore be most simply reduced by dispensing with lifetime measures and concentrating on point- and period-prevalence. Since, however, the comments of Regier et al (1998) already mentioned refer explicitly to one-year estimates, this improvement alone would not resolve the basic problem.

\section{Identifying clinical syndromes in community populations}

Psychiatric symptoms reported by fieldstudy respondents can be analysed to see how frequently they occur in combinations resembling the distinctive syndromes of hospital-treated patients, and hence to gauge the confidence with which individuals can be assigned to diagnostic categories such as schizophrenia, depressive disorder or anxiety state. This approach, exemplified by the 'Index of Definition', which Wing and his colleagues developed in conjunction with the PSE-CATEGO classification system (Wing et al, 1978), has yielded fairly conservative prevalence estimates in community surveys (Bebbington, 1990). Diagnostic confidence cannot, however, be equated with clinical severity or need for treatment. Indeed, Wing (1976) was careful to stress that non-specific symptoms such as worrying, sleeplessness and irritability, although not of much help in differential diagnosis, may be very severe in terms of personal distress or social impairment. Hence, an undeniable need for medical treatment can exist among many persons who score below threshold on the Index of Definition.

\section{Adding criteria for severity and functional impairment}

Regier et al (1998), arguing that selfreported symptoms alone are inadequate as case criteria, recommend that these should be supplemented by data on illness severity and duration, comorbidity and associated functional impairment. If such variables, as well as the clinical symptoms of illness, could be rated reliably at a single interview, this would constitute a real advance in public-health knowledge. There are, however, no grounds a priori to suppose that such information is any simpler to obtain than that relating to diagnosis. In particular the rating of functional impairments and disabilities, which lies at the core of individual needs-assessment, calls for detailed inquiry and, in respect of those with severe mental illness, for questioning of health care professionals or other informants (Brewin \& Wing, 1993; Phelan et al, 1995). The proposal thus raises questions with regard to research manpower and costs, as well as to respondents' adherence.

\section{Two-stage procedures of case ascertainment}

The solution preferred by Dohrenwend (1990) is a two-stage procedure, in which initial screening by means of a short rating scale is followed by a more detailed examination of all persons with abnormal scores, to weed out 'false positives'. This approach, in his view:

"... capitalizes on the ability of a psychometric in-
strument to provide reliable measurement over
the full range of dimensions of psychopathology
and on the ability of a clinical examination to pro-
vide reliable diagnoses in groups where the types
of symptomatology are not rare" (Dohrenwend,
1990).

The point is well taken, and indeed the two-stage approach is now firmly established in psychiatric epidemiology. One cannot, however, assume that it will sharpen the focus on persons with the greatest need for treatment. In the Israeli survey that Dohrenwend cites as an example, nearly half a cohort of adults were rated positively at first-stage screening, and after interview by psychiatrists using a short form of the Schedule for Affective Disorders and Schizophrenia (SADS-L) about one-fifth were judged to meet Research Diagnostic Criteria (RDC) for "a disorder at the definite level" (Levav et al, 1993). Making allowance for age differences, this result is not much different from those of the ECA and NCS projects. Even community surveys in which a more rigorous second-stage examination such as SCAN was used have indicated levels of treatment need in populations on a scale that could not be met by specialist services (Bebbington et al, 1997).

\section{Restoring a place for clinical judgement}

It is not generally appreciated how far most of the standardised interviews have diverged from clinical 'case taking' methods in psychiatry. As set out, for example, in the Maudsley Handbook of Practical Psychiatry (Goldberg, 1997), these do not include systematic checking of a symptom inventory, but start from the presenting condition, and proceed to assessment of the patient's mental status from his or her appearance and general behaviour, talk, mood, attention and thought content; any evidence suggesting abnormal beliefs, interpretation of events or experiences referred to one's environment, body or person, and the cognitive status in terms of memory, orientation and general knowledge. 
A number of the earlier semi-structured interviews, including the Mental Status Schedule (Spitzer et al, 1964) and the Clinical Interview Schedule (CIS; Goldberg et $a l, 1970)$ did respect the need for reported symptoms and abnormalities observed at interview to be rated separately, but in the 1980s this distinction was largely abandoned as reliance on lay interviewers increased. Thus, a revision of the CIS at that time was undertaken on the grounds that:

“. . . ratings requiring clinical judgement add little information to those based on self-report, may be less reliable and may lead to the biassed assessment of anxiety and depression" (Lewis \& Williams, 1989).

This conclusion, being based not on psychiatric material, but on various samples (general practice patients, antenatal clinical attenders, people with diabetes and Home Office civil servants) whose mean symptom scores were all low, cannot be generalised to cases of serious mental disorder. There is now growing agreement that manifest abnormalities should also be rated, although not as to the best way of going about it. Brugha et al (1999a), from their experience with SCAN, argue that diagnostic validity can be ensured in large-scale surveys if non-medical interviewers are trained to make basic clinical judgements, but how successfully this can be achieved within a brief period is as yet unclear. Kessler (1999), working with the CIDI, has proposed a number of strategies including use of audio-tapes and correction of interview ratings by means of comparison with a sofar undefined clinical 'gold standard'.

\section{COVERAGE OF SEVERE MENTAL DISORDERS}

Community prevalence surveys have proved most effective in the enumeration of common, non-psychotic conditions; notably, anxiety and depressive states and their congeners among adults, learning disability and behavioural disorders in school-age children and cognitive impairment in the elderly. Their contribution to the epidemiology of psychotic illness, in contrast, has been distinctly modest. Low base rates in the general population, together with problems of non-response and difficulties in contacting high-risk groups of homeless persons and transients, dictate that case numbers will be small, and frequency estimates unreliable, except in the most ambitious projects. In area surveys listed by Goldberg \& Huxley (1992) as having employed standardised diagnostic interviews, some 250 cases of functional psychosis (schizophrenia, paranoid states and bipolar affective disorders) were found among a total of 23000 respondents who could be fully examined, the mean one-month prevalence rate being 11 per 1000 . Such low case-yields imply that community surveys are an uneconomical way to study the psychoses, unless their detection is in effect a 'spin off' from the enumeration of common, non-psychotic syndromes - in which case prevalence estimates are likely to be inaccurate.

One way of resolving the dilemma might be to undertake a first-stage screening targeted on the psychoses, and thus to reduce the numbers requiring a full diagnostic assessment. The British Psychiatric Morbidity Survey (Jenkins et al, 1998) employed in its household sampling survey a battery of items from the Psychosis Screening Questionnaire (PSQ), as well as collecting information from key informants (community mental health teams and general practitioners (GPs)) on persons with psychotic illness known to them. On previous testing the PSQ had performed extremely well, with a sensitivity of $96.9 \%$ and a specificity of $95.3 \%$ (Bebbington \& Nayani, 1995). Since, however, the positive predictive value of a test is directly correlated with prevalence of the target group, it will tend to be low for rare disorders. To find each case of psychosis, assuming the screen maintained its performance level under field-study conditions, an average of six screen-positives would have to be examined if the true prevalence were $1 \%$, but many more given the oneyear prevalence of 4.4 per 1000 estimated in the household survey, and since many of these cases were identified by respondents' self-reports of mental illness or antipsychotic medication, rather than by screening for psychotic symptoms (H. Meltzer, personal communication, 1999). Such a result might still signify advantage over a single-stage design (Shrout \& Newman, 1989 ) in a project concerned only with psychoses, but as in practice these disorders are almost always studied jointly with more common conditions, comparing the relative efficiency of one- and two-stage survey designs becomes a complex task.

Much larger case numbers, and hence more robust and reliable prevalence estimates, have come from key-informant studies of cases of psychosis known to local service agencies; whether only the specialist services covered by cumulative case registers
(Freeman \& Alpert, 1986), or all relevant medical and social agencies co-opted as information sources in ad hoc surveys (Jeffreys et al, 1997). Informant surveys relying on second-hand data cannot, however, resolve all questions of method. Problems of clinical validity will remain, while a variable proportion of cases present in the population will be unknown to, or out of contact with, all service agencies, and identifiable only with the aid of special searches (Von Korff et al, 1985).

A systematic attempt to overcome these difficulties was made as part of the Australian National Survey of Mental Health and Wellbeing, in which a key informant study of 'low prevalence' disorders (i.e. functional psychoses) in four widely separated, predominantly urban areas was carried out independently of the main community-sample survey (Australian Bureau of Statistics, 1999). All individuals in contact with 'mainstream' mental health services were examined using an instrument (Diagnostic Interview for Psychosis; Jablensky et al, 1999) specially developed for the survey and, in addition, persons with suspected psychotic illness were screened by the same technique in hostels, boarding houses, drop-in centres, refuges and shelters, as well as by office psychiatrists and GPs who agreed to participate.

According to the recent report (Jablensky et al, 1999), the prevalence of psychotic illness among adults in these areas lay in the range 4-7 per 1000 with a weighted mean of 4.7 per $1000,60 \%$ of cases being accounted for by schizophrenia. Of a total of 980 persons in contact with service agencies during the census month, $26.7 \%$ were identified in psychiatric in-patient units, $43.4 \%$ in psychiatric out-patient departments or community clinics, $8.9 \%$ by private psychiatrists, $8.8 \%$ by GPs, and $12.2 \%$ in hostels, shelters for the homeless or other 'marginal' sites. An additional 146 persons were identified who were known to services but had not made contact in the census month. Since the parallel nationwide community survey employed both a different sampling method and different instruments, and moreover did not attempt to cover all diagnostic categories (Australian Bureau of Statistics, 1998), it is hardly possible to estimate what proportion of 'low prevalence' disorders could have been picked up by direct population screening. How the results of these different research strategies can best be combined to yield integrated prevalence estimates for 
both low-prevalence and common mental disorders remains an open question.

\section{IMPLICATIONS OF GENUINELY HIGH PSYCHIATRIC PREVALENCE}

Many who agree with Regier and his colleagues, that psychiatric field studies should in future pay closer regard to the realities of health care provision and unmet need, may baulk at the implied corollary that research findings should be constrained by the straitjacket of 'medical necessity' as this is interpreted in health insurance schemes and managed care programmes. Epidemiology, after all, is a public health discipline, not an instrument of cost-containment. Distress and functional impairment are widespread among psychologically-disturbed individuals who would not satisfy the criteria imposed to justify specialist treatment costs, yet none the less require medical help.

A better basis for service planning may be a needs-assessment approach in which the appropriate care-packages can be specified in terms of psychiatric in-patient or extramural treatment, community support, primary health care, or some combination of these. The hierarchy of 'levels and filters' proposed by Goldberg \& Huxley (1992) derives from a model in which referral to specialist services is usually via the GP, and the term filter refers to each of the selective stages at which a person in the community (Level 1), consults his or her doctor (Level 2), the doctor recognises a mental disturbance (Level 3), the patient may then be referred to a psychiatric agency (Level 4) and, if necessary, admitted to in-patient care (Level 5).

That a majority of psychiatric disorders at any point in time will be under the sole care of GPs was demonstrated in the UK over 30 years ago (Shepherd et al, 1966), and has since been substantiated in many other Western countries (Cooper \& Eastwood, 1992; Üstün \& Sartorius, 1995). Goldberg \& Huxley (1992), using compiled research data, estimated the one-year prevalence of 'conspicuous' cases (i.e. those identified by doctors) as 101.5 per 1000 , and contrasted this rate with the 23.5 per 1000 referred to mental health services. Even in the USA, general practice forms a major part of the de facto mental health system, providing care each year for an estimated $43 \%$ of people with mental or addictive disorders compared with $40 \%$ treated by specialist agencies (Regier et al,
1993). High total prevalence estimates may thus have stronger implications for the primary health care system than for specialist services. Treatment and management of most common mental disorders is best regarded as an integral part of the continuing, whole-patient care provided by the general practice team.

If many cases diagnosed in community surveys were actually transient, homoeostatic reactions, as suggested by Regier et al (1998), one would expect to find these mainly represented at the lower levels of the model. Goldberg \& Huxley (1992) noted inferential evidence for a high rate of shortterm, apparently spontaneous remission in both community and general practice studies, but commented that this might be partly explained by false-positive results at initial screening. Longer-term follow-up is in any case necessary to examine the patterns of temporary remission and recurrence among persons who are vulnerable to minor stress.

Cooper et al (1969), in a generalpractice study, found that of patients who consulted with new psychiatric episodes during one year, a steady proportion presented with similar complaints in each of the five subsequent years. Forty-eight per cent were treated for psychiatric illness in two or more of these five years, and only $28 \%$ remained apparently well for the whole period. This proportion represented in effect the maximum for new cases with complete and lasting remission, since some of the patients concerned might have experienced further episodes that were not seen, or not diagnosed, by the practice doctors. Discarding $28 \%$ of the Goldberg \& Huxley 'conspicuous morbidity' estimate would still leave an annual rate of 73 patients per 1000 with chronic or recurrent conditions, of whom on average only onethird would be referred to mental health agencies each year. In addition, a substantial proportion of unrecognised Level 2 cases might benefit from appropriate treatment, although it seems that improving medical recognition of psychological disorders does not by itself ensure a better outcome (Tiemens et al, 1996).

\section{DISCUSSION}

Although the justification for descriptive prevalence surveys of mental illness must ultimately rest on their utility for health care policy and planning, third-generation survey researchers in their pursuit of formal reliability have long marched to a different drummer. A careful reappraisal is now called for to achieve a better balance of priorities in the future. Before embarking on new large-scale projects, it would seem prudent at least to explore ways of ensuring greater clinical and public health relevance for the research findings. The various proposals for improving case-assessment procedures outlined above deserve systematic testing.

Yet refinement of the standardised techniques alone may not be sufficient: the underlying assumption that mental health care needs can be satisfactorily gauged by community surveys, designed to net all ICD- or DSM-classified conditions, is itself open to challenge. The complementary roles of population-based case registers, key-informant surveys and general practice studies should also be carefully assessed. If service agency collaboration can be most readily secured at local area level, not least because of data protection requirements, a question arises with regard to the relative merits of national population surveys and alternative research designs, such as investigation of selected, contrasting area populations. The latter approach, already employed in the ECA Study and the Australian low prevalence survey, has the potential advantage that it can provide a starting point for population-based evaluative research.

To link epidemiology to controlled intervention studies an appropriate service framework is necessary, and in many societies the most promising is that of primary health care. But because the milder forms of mental disorder fit a dimensional model at least as readily as a categorical one (Goldberg \& Huxley, 1992), a rigid dichotomy into cases and normals could here be inappropriate, and even detrimental in situations where health insurers are seeking to 'carve out' the mental health component from general medical cover. If a monaxial disease classification cannot do justice to the close intertwining of physical and mental ill-health so often encountered in general practice (Crombie, 1963), the working definition of prevalence may have to be adapted to suit a bi- or multiaxial classification of health problems. That, however, is a matter to be decided on clinical and scientific grounds, not simply in conformity with managed care plans.

\section{ACKNOWLEDGEMENTS}

We are grateful to Scott Henderson and Ailsa Korten, Psychiatric Epidemiology Research Centre, 
Canberra, for supplying material from the Australian National Survey of Mental Health and Wellbeing, and Howard Meltzer, Office for National Statistics Social Survey Division, London, for information on the British National Psychiatric Morbidity Survey.

\section{REFERENCES}

American Psychiatric Association (1994) Diagnostic and Statistical Manual of Mental Disorders (4th edn) (DSM-IV). Washington, DC: APA

Anthony, J. C., Folstein, M., Romanowski, A. J., et al (1985) Comparison of the lay diagnostic interview schedule and a standardized psychiatric diagosis: experience in Eastern Baltimore. Archives of General Psychiatry, 42, 667-675.

Australian Bureau of Statistics (1998) Mental Health and Wellbeing Profile of Adults. Canberra: ABS.

- (1999) People with Psychotic Illness - A Report from the National Survey of Mental Health and Wellbeing, Australia 1997-1998. Report 3. Canberra: ABS.

Bebbington, P. E. (1990) Population surveys of psychiatric disorder and the need for treatment. Social Psychiatry and Psychiatric Epidemiology, 25, 33-40.

— \& Nayani, T. (1995) The Psychosis Screening Questionnaire. International Journal of Methods in Psychiatric Research, 5, II-19.

_, Marsden, L. \& Brewin, C. R. (1997) The need for psychiatric treatment in the general population: the Camberwell Needs for Care Survey. Psychological Medicine, 27, 82I-834.

Brewin, C. \& Wing, J. K. (1993) The MRC Needs for Care Assessment: progress and controversies. Psychological Medicine, 23, 837-84I.

Brugha, T. S., Bebbington, P. \& Jenkins, R. (1999a) A difference that matters: comparisons of structured and semi-structured psychiatric diagnostic interviews in the general population. Psychological Medicine, 29, 1013-1020.

_ , Nienhuis, F. J., Bagchi, D., et al (1999b) The survey form of SCAN: the feasibility of using experienced lay survey interviewers to administer a semi-structured systematic clinical assessment of psychotic and non-psychotic disorders. Psychological Medicine, 29, 703-7।I.

Cooper, B., Fry, J. \& Kalton, G. (1969) A longitudinal study of psychiatric morbidity in a general practice population. British Journal of Preventive and Social Medicine, 23, 210-217.

— \& Eastwood, R. (eds) (1992) Primary Health Care and Psychiatric Epidemiology. London: Tavistock.

Crombie, D. L. (1963) The Procrustean bed of medical nomenclature. Lancet, i, 1205-1206.

Dohrenwend, B. P. (1990) "The problem of validity in field studies of psychological disorders" revisited. Psychological Medicine, 20, 195-208.

— \& Dohrenwend, B. S. (1974) Social and cultural influences on psychopathology. Annual Review of Psychology, 25, 4I7-452.

Ford, W. E. (1998) Medical necessity: its impact in managed mental health care. Psychiatric Services, 49, 183-184.

Freeman, H. \& Alpert, M. (1986) Prevalence of schizophrenia in an urban population. British Journal of Psychiatry, 149, 603-6II.

Goldberg, D. (ed.) (1997) The Maudsley Handbook of Practical Psychiatry. Oxford: Oxford University Press.

BRIAN COOPER, FRCPsych, Honorary Research Fellow, Institute of Psychiatry, London; BRUCE SINGH, FRANZCP, Cato Professor and Head of Department, Department of Psychiatry, University of Melbourne, Victoria, Australia

Correspondence: Dr Brian Cooper, Section of Old Age Psychiatry, Institute of Psychiatry, De Crespigny Park, Denmark Hill, London SE5 8AF

(First received 20 August 1999, final revision 15 October 1999, accepted I5 October 1999)

\section{_, Cooper, B., Eastwood, M. R., et al (1970) A}

standardised psychiatric interview for use in community surveys. British Journal of Preventive and Social Medicine, 24, 18-23.

— \& Huxley, P. (1992) Common Mental Disorders: a Bio-Social Model. London: Routledge.

Helzer, J. E., Robins, L. N., McEvoy, L. T., et al (1985) A comparison of clinical and diagnostic interview schedule diagnoses. Physician re-examination of layinterviewed cases in the general population. Archives of General Psychiatry, 42, 657-666.

Jablensky, A., McGrath, J., Herrman, H., et al (1999) People Living with Psychotic Illness: An Australian Study. National Survey of Mental Health and Wellbeing, Report 4. Canberra: Department of Health and Aged Care.

Jeffreys, S. E., Harvey, C. A., McNaught, A. S., et al (1997) The Hampstead Schizophrenia Survey 1991. I: Prevalence and service use comparisons in an inner London health authority, 1986-1991. British Journal of Psychiatry, I70, 301-306.

Jenkins, R., Bebbington, P., Brugha, T. S., et al (1998) British psychiatric morbidity survey. British journal of Psychiatry, 173, 4-7.

Kendler, K. S., Gallagher, T. J., Abelson, J. M., et al (1996) Lifetime prevalence, demographic risk factors, and diagnostic validity of non-affective psychosis as assessed in a US community sample. The National Comorbidity Survey. Archives of General Psychiatry, 53, 1022-1031.

Kessler, R. C. (1999) The World Health Organization International Consortium on Psychiatric Epidemiology (ICPE): initial work and future directions. Acto

Psychiatrica Scandinavica, 99, 2-9.

Levav, I., Kohn, R., Dohrenwend, B. P., et al (1993) An epidemiological study of mental disorders in a 10 -year cohort of young adults in Israel. Psychological Medicine, 23, 691-707.

Lewis, G. \& Williams, P. (1989) Clinical judgement and the standardized interview in psychiatry. Psychological Medicine, 19, 97I-979.

_, Pelosi, A. J., Araya, R., et al (1992) Measuring psychiatric disorder in the community: a standardized assessment for use by lay interviewers. Psychological Medicine, 22, 465-486.

National Advisory Mental Health Council (1993) Health care reform for Americans with severe mental illnesses. American Journal of Psychiatry, 150, 1447-1465.

Parker, G. (1987) Are lifetime prevalence estimates in the ECA study accurate? Psychological Medicine, I7, 275-282.

Phelan, M., Slade, M., Thornicroft, G., et al (1995) The Camberwell Assessment of Need: the validity and reliability of an instrument to assess the needs of people with severe mental illness. British Journal of Psychiatry, 167, 589-595.

Regier, D. A., Narrow, W. E. \& Rae, D. S. (1993) The de facto US mental and addictive disorders service system: Epidemiologic Catchment Area prospective one-year prevalence rates of disorders and services. Archives of General Psychiatry, 50, 85-94.
_, Kaelber, C. I., Rae, D. S., et al (1998) Limitations of diagnostic criteria and assessment instruments for mental disorders. Archives of General Psychiatry. 55, 109-115.

Robins, L. N., Helzer, J. E., Croughlan, J., et al (198I) The NIMH Diagnostic Interview Schedule: its history, characteristics and validity. Archives of General Psychiatry, 38, $381-389$

_ , Wing, J. K., Wittchen, H. U., et al (1988) The Composite International Diagnostic Interview. An epidemiologic instrument suitable for use in conjunction with different diagnostic systems and in different cultures. Archives of General Psychiatry, 45, 1069-1077.

Rodgers, B. \& Mann, S. A. (1986) The reliability and validity of PSE assessment by lay interviewers: a national population survey. Psychological Medicine, 16, 689-700.

Shepherd, M., Cooper, B., Brown, A. C., et al (1966) Psychiatric Illness in General Practice. London: Oxford University Press.

Shrout, P. E. \& Newman, S. C. (1989) Design of twophase prevalence surveys of rare disorders. Biometrics, 45, 549-555.

Spitzer, R. L., Fleiss, J. L. \& Burdock, E. L., et al (1964) The Mental Status Schedule: rationale, reliability and validity. Comprehensive Psychiatry, 5, 384-395.

_, Endicott, J., Fleiss, J. L., et al (1970) The Psychiatric Status Schedule: a technique for evaluating psychopathology and impairment in role functioning. Archives of General Psychiatry, 23, 4I-55.

Tiemens, B. G., Ormel, J. \& Simon, G. E. (1996) Occurrence, recognition and outcome of psychological disorders in primary care. American Journal of Psychiatry, 153, 636-644

Üstün, T. B. \& Sartorius, N. (eds) (1995) Mental Illness in General Health Care. An International Study. Chichester: J.Wiley

Von Korff, M., Nestadt, G., Romanski, A., et al (1985) Prevalence of treated and untreated DSM-III schizophrenia. Results of a two-stage community survey. Journal of Nervous and Mental Disease, 173, 577-58I.

Wing, J. K. (1976) A technique for studying psychiatric morbidity in in-patient and out-patient series and in general population samples. Psychological Medicine, 6 , 665-67l.

_ , Cooper, J. E. \& Sartorius, N. (1974) The Measurement and Classification of Psychiatric Symptoms. Cambridge: Cambridge University Press.

_ , Mann, S. A., Leff, J. P., et al (1978) The concept of a 'case' in psychiatric population surveys. Psychological Medicine, 8, 203-217.

— , Bebbington, P. E. \& Robins, L. N. (1981) What is Case? The Problem of Definition in Community Surveys. London: Mclntyre.

_, Babor, T., Brugha, T., et al (1990) SCAN

Schedules for Clinical Assessment in Neuropsychiatry Archives of General Psychiatry, 47, 589-593.

World Health Organization (1993) The ICD-10 Classification of Mental and Behavioural Disorders: Diagnostic Criteria for Research. Geneva: WHO. 